\title{
ISOMORPHISMS OF LOCALLY COMPACT GROUPS AND BANACH ALGEBRAS
}

\author{
ANTHONY TO-MING LAU ${ }^{1}$ AND KELLY MCKENNON
}

\begin{abstract}
If $G$ is a locally compact group, then $\operatorname{UBC}_{r}(G)^{*}$, the dual of the space of bounded right uniformly continuous complex-valued functions on $G$, with the Arens product is a Banach algebra. We prove in this paper a result that will have as a consequence the following: Let $G_{1}, G_{2}$ be locally compact groups. Then the Banach algebras $\mathrm{UBC}_{r}\left(G_{1}\right)^{*}$ and $\mathrm{UBC}_{r}\left(G_{2}\right)^{*}$ are isometric isomorphic if and only if $G_{1}$ and $G_{2}$ are topologically isomorphic.
\end{abstract}

1. Introduction. J. Wendel proved in [7] that if $G_{1}$ and $G_{2}$ are locally compact groups such that the group algebras $L_{1}\left(G_{1}\right)$ and $L_{1}\left(G_{2}\right)$ are isometric isomorphic, then $G_{1}$ and $G_{2}$ are topologically isomorphic. Later B. Johnson [3] proved that the same conclusion holds when the group algebras are replaced by the measure algebras $M\left(G_{1}\right), M\left(G_{2}\right)$ of $G_{1}$ and $G_{2}$ respectively.

Let $\mathrm{UBC}_{r}(G)$ denote the Banach space of bounded right uniformly continuous complex-valued functions on a locally compact group $G$ (see $[4$, p. 275]) with the supremum norm. On the dual Banach space, $\operatorname{UBC}_{r}(G)^{*}$, we may define a product by $\langle m \odot n, f\rangle=\left\langle m, n_{l}(f)\right\rangle$, where $n_{l}(f)(x)=\left\langle n, l_{x} f\right\rangle, l_{x} f(y)=f(x y)$, for any $m, n$ $\in \mathrm{UBC}_{r}(G)^{*}, f \in \operatorname{UBC}_{r}(G)$, and $x, y \in G$. Then $\operatorname{UBC}_{r}(G)^{*}$ with respect to this product is a Banach algebra (see [4, p. 275]). In this paper we shall prove a theorem (Theorem 1) which will have as consequences both Johnson's result [3, Corollary 1] and the following:

TheORem 2. Let $G_{1}, G_{2}$ be locally compact groups. Then the Banach algebras $\mathrm{UBC}_{r}\left(G_{1}\right)^{*}$ and $\operatorname{UBC}_{r}\left(G_{2}\right)^{*}$ are isometric isomorphic if and only if $G_{1}$ and $G_{2}$ are topologically isomorphic.

2. Some technical lemmas. Throughout this section, $G$ will denote a locally compact group with a fixed left Haar measure $\lambda$. Integration with respect to $\lambda$ will be denoted by $\int_{G} \ldots d x$. If $f, h$ are complex-valued measurable functions defined $\lambda$ a.e. on $G$, let

$$
\begin{aligned}
\tilde{f}(x) & =f\left(x^{-1}\right), \quad l_{x} f(y)=f(x y), \quad r_{x} f(y)=f(y x), \\
(f * h)(x) & =\int_{G} f(y) h\left(y^{-1} x\right) d y, \quad(\mu * h)(x)=\int_{G} h\left(y^{-1} x\right) d \mu(y)
\end{aligned}
$$

Received by the editors May 31, 1979.

AMS (MOS) subject classifications (1970). Primary 43A10, 43A20; Secondary 43A60.

Key words and phrases. Locally compact group, group algebra, measure algebra, Arens product, isometric isomorphism, topological isomorphism, uniformly continuous function, almost periodic function.

${ }^{1}$ The first author is supported by NRC grant A7679. 
for any $x, y \in G$ and regular Borel measure $\mu$ on $G$ whenever the formula makes sense.

Let $C(G)$ denote the space of bounded continuous complex-valued functions on $G$ with the supremum norm $\|\cdot\|_{u}$, and let $C_{0}(G)$ be the closed subspace of $C(G)$ consisting of all functions vanishing at infinity. A closed linear subspace $X$ of $C(G)$ is left introverted (see Day [1, p. 540]) if $l_{a}(X) \subseteq X$ for each $a \in G$, and for each $m \in X^{*}, f \in X$, the function $m_{l}(f)$ on $G$ defined by $m_{l}(f)(x)=m\left(l_{x} f\right), x \in G$, is also in $X$. In this case, the Arens multiplication on $X^{*}$ defined by $\langle n \odot m, f\rangle=$ $\left\langle n, m_{l}(f)\right\rangle$ for each $f \in X, n, m \in X^{*}$ makes sense. Furthermore, $X^{*}$ with this multiplication is a Banach algebra (see [1, §6]). Examples of left introverted subspaces of $C(G)$ include $C_{0}(G), \mathrm{UBC}_{r}(G)$, and the space of almost periodic (resp. weakly almost periodic) continuous functions on $G$, denoted by $\operatorname{AP}(G)$ (resp. WAP $(G)$ ). In the case of $C_{0}(G)^{*}=M(G)$, the multiplication $\odot$ on $M(G)$ is precisely the convolution of measures as defined in $[4$, p. 266].

If $a \in G, \delta_{a}$ will denote either the point-measure at $a$ in $M(G)$, or the pointevaluation linear functional in $X^{*}$ when $X$ is a subspace of $C(G)$.

LemMa 1. Let $m \in C_{0}(G)^{*}$. Then $m$ has a unique norm preserving extension to a continuous linear functional on $C(G)$.

Proof. We may assume that $\|m\|=1$. Let $\mu$ be a regular Borel measure on $G$ such that $m(f)=\int_{G} f(x) d \mu(x)$ for each $f \in C_{0}(G)$. Define $\tilde{m}(f)=\int_{G} f(x) d \mu(x)$ for all $f \in C(G)$. Then $\tilde{m} \in C(G)^{*}, \tilde{m}$ extends $m$, and $\|\tilde{m}\|=1$.

If $n \in C(G)^{*}$ is another extension of $m$, and $\|n\|=1$, let $F$ denote the set of linear combinations of point evaluations on $G$ in the unit ball of $C(G)^{*}$. If $n$ is not in the weak*-closure of $F$, then by [2, p. 417, Theorem 10] there exist $f \in C(G)$, a constant $c$ and $\varepsilon>0$ such that

$$
\operatorname{Re}\langle f, \phi\rangle \leqslant c-\varepsilon \leqslant c \leqslant \operatorname{Re}\langle f, n\rangle
$$

for all $\phi \in F$. Now if $a \in G$, and $f(a)=r e^{i \theta}$, let $\phi=e^{-i \theta} \delta_{a}$; then $\langle\phi, f\rangle=r<$ $c-\varepsilon$. Hence $|f(a)|=r \leqslant c-\varepsilon$. In particular, $\|f\|_{u} \leqslant c-\varepsilon$.

However $c \leqslant \operatorname{Re}\langle f, n\rangle \leqslant|\langle f, n\rangle| \leqslant\|n\|\|f\|_{u} \leqslant c-\varepsilon$ which is impossible. Hence $n$ is in the weak ${ }^{*}$-closure of $F$.

Let $m_{\alpha}$ be a net in $F$, such that $m_{\alpha}(f) \rightarrow n(f)$ for each $f \in C(G)$. Let $q\left(m_{\alpha}\right)$ denote the restriction of $m_{\alpha}$ to $C_{0}(G)$. Then $\left\|q\left(m_{\alpha}\right)\right\| \leqslant 1$, and $q\left(m_{\alpha}\right)(f) \rightarrow \int_{G} f d \mu$ for each $f \in C_{0}(G)$. Hence $\left\|q\left(m_{\alpha}\right)\right\| \rightarrow\|\mu\|=1$. It follows from [6, Theorem 3.9] that $\left\langle m_{\alpha}, f\right\rangle$ also converges to $\int_{G} f d \mu=\tilde{m}(f)$ for each $f \in C(G)$. In particular, $n=\tilde{m}$.

LEMMA 2. Let $X$ be a left introverted closed subspace of $C(G)$ containing $C_{0}(G)$. If $m \in X^{*}$ such that $m_{l}: X \rightarrow X$ is an isometry, then there exist $x \in G$ and $\lambda \in C$, $|\lambda|=1$ such that $m_{l}(f)=\lambda r_{x}(f)$ for all $f \in X$.

Proof. We first assume that $X=C_{0}(G)$. Let $\mu \in M(G)$ such that $\langle m, f\rangle=$ $\int_{G} f d \mu$ for all $f \in C_{0}(G)$.

Define: $\tau: L_{1}(G) \rightarrow L_{1}(G)$ by $\tau(f)=\mu * f, f \in L_{1}(G)$. Then $\tau$ is an isometry. Clearly $\|\tau(f)\|_{1} \leqslant\|f\|_{1}$ for each $f \in L_{1}(G)$ since $\|\mu\|=1$. To prove the reverse 
inequality, let $f \in L_{1}(G)$ be fixed. For any $\varepsilon>0$, choose $h \in C_{0}(G)$ such that $\|h\|_{u}=1$ and $\left|\int f(x) h(x) d x\right|>\|f\|_{1}-\varepsilon$. Then $\|(f * \tilde{h}) \sim\|_{u}>\|f\|_{1}-\varepsilon$. Hence

$$
\begin{aligned}
\|\mu * f\|_{1} & =\|\mu * f\|_{1}\|\tilde{h}\|_{u} \geqslant\|(\mu * f) * \tilde{h}\|_{u} \\
& =\|\mu *[(f * \tilde{h}) \sim] \sim\|_{u}=\left\|m_{l}((f * \tilde{h}) \sim)\right\|_{u} \\
& =\|(f * \tilde{h}) \sim\|_{u}>\|f\|_{1}-\varepsilon .
\end{aligned}
$$

By [7, Theorem 3], there exist $y \in G$ and $\lambda \in C,|\lambda|=1$, such that $\mu * f=\lambda l_{y} f$ for all $f \in L_{1}(G)$. In particular $m_{l}(f)=(\mu * \tilde{f})^{\sim}=\lambda r_{x} f$, where $x=y^{-1}$, for all $f \in$ $C(G)$ with compact support. Hence $m_{l}(f)=\lambda r_{x}(f)$ for all $f \in C_{0}(G)$.

The general case follows from Lemma 1 by considering the restriction of $m$ to $C_{0}(G)$.

If $X$ is a linear subspace of $C(X)$, and $h \in L_{1}(G)$, we define $\tau(h) \in X^{*}$ by

$$
\tau(h)(f)=\int_{G} f(x) h(x) d x \quad \text { for all } f \in X .
$$

LEMMA 3. If $X$ is a closed left introverted subspace of $C(X)$ consisting of left uniformly continuous functions, then the set

$$
\left\{m_{l}(f) ; m=\tau(h), h \in L_{1}(G) \text { and } f \in X\right\}
$$

is norm dense in $X$.

Proof. Let $\left\{h_{\alpha}\right\}$ be a bounded approximate identity in $L_{1}(G)$ and $m_{\alpha}=m\left(h_{\alpha}\right)$. Then

$$
\left\|\left(m_{\alpha}\right)_{l}(f)-f\right\|_{u}=\left\|\left(h_{\alpha} * \tilde{f}\right)^{\sim}-f\right\|_{u}=\left\|h_{\alpha} * f-f\right\|_{u}
$$

which converges to zero (see $[5,32.48])$.

REMARK. Lemma 3 is false when the functions in $X$ are not left uniformly continuous. For example, if $X=\operatorname{UBC}_{r}(G)$, then for each $f \in X, h \in L_{1}(G)$, and $m=\tau(h)$, the function $m_{l}(f)=f * \tilde{h}$ is both left and right uniformly continuous (see $[4,20.16])$.

3. The main theorem. We are now ready to prove our main result.

THEOREM 1. Let $G_{1}, G_{2}$ be locally compact groups. Let $X_{i}, i=1,2$, be a closed left introverted subspace of $C\left(G_{i}\right)$ containing $C_{0}\left(G_{i}\right)$. If the Banach algebras $X_{1}^{*}$ and $X_{2}^{*}$ are isometric isomorphic, then $G_{1}$ and $G_{2}$ are topologically isomorphic.

Proof. Let $\beta: X_{1}^{*} \rightarrow X_{2}^{*}$ be an isometric isomorphism. For $a \in G$ and $m=\delta_{a}$, we have $m^{-1}=\delta_{b}$ in $X_{1}^{*}$, where $b=a^{-1}$, and so

$$
\|\beta(m)\|=\|m\|=1=\left\|m^{-1}\right\|=\left\|\beta(m)^{-1}\right\|
$$

which implies that $\beta(m)$ is a linear isometry. An application of Lemma 2 shows that there exist $\gamma(a)$ in $G_{2}$ and $\lambda(a) \in C$ such that $\beta(m)_{l}=\lambda(a) r_{\gamma(a)}$. Then as readily checked, $\gamma$ is an algebraic isomorphism from $G_{1}$ onto $G_{2}$. It remains to show that $\gamma$ is a homeomorphism. 
Let $\left\{x_{\alpha}\right\}$ be a net in $G_{1}$ converging to the identity $e_{1}$. Let $\left\{\gamma\left(x_{\pi}\right)\right\}$ be any subnet of the net $\left\{\gamma\left(x_{\alpha}\right)\right\}$. By Alaoglu's theorem, we may assume (passing to a subnet if necessary) that the net $\theta_{\pi}=\beta\left(\delta_{x_{n}}\right)$ converges to some $\eta \in X_{2}^{*}$ in the weak*-topology of $X_{2}^{*}$. Since the net $\left\{\lambda\left(x_{\pi}\right)\right\}$ is bounded, we may further assume that the net $\lambda\left(x_{\pi}\right)$ also converges. Let $h \in L_{1}(G)$, and $m=\tau(h)$ (see Lemma 3), then

$$
\left(\delta_{x_{*}} \odot m\right)(f)=\int_{G_{1}} h\left(x_{\pi}^{-1} y\right) f(y) d y
$$

for each $f \in X_{1}$. Hence $\left\|\delta_{x_{\gamma}} \odot m-m\right\|<\left\|l_{z_{\pi}} h-h\right\|_{1}$, where $z_{\pi}=x_{\pi}^{-1}$, which converges to zero. This implies that the net $\left\|\theta_{\pi} \odot \beta(m)-\beta(m)\right\| \rightarrow 0$ also. In particular,

$$
[\beta(m)](k)=\lim _{\pi} \theta_{\pi} \odot \beta(m)(k)=\eta \odot \beta(m)(k)
$$

for each $k \in X_{2}$. So $\beta(m)=\eta \odot \beta(m)$ and hence $m=\beta^{-1}(\eta) \odot m$. Consequently $m_{l}(f)\left(e_{1}\right)=\beta^{-1}(\eta)\left(m_{l}(f)\right)$ for each $f \in X_{1}$ and $m=\tau(h), h \in L_{1}(G)$. Since $C_{0}\left(G_{1}\right)$ $\subseteq X_{1}$ and is left introverted, Lemma 3 implies that $\beta^{-1}(\eta)$ agrees with $\delta\left(e_{1}\right)$ on $C_{0}\left(G_{1}\right)$. Since $1=\left\|\delta_{e_{1}}\right\| \leqslant\left\|\beta^{-1}(\eta)\right\|=\|\eta\| \leqslant 1$, it follows from Lemma 2 that $\beta^{-1}(\eta)$ and $\delta_{e_{1}}$ agree on $X_{1}$ also. In particular $\eta=\delta_{e_{2}}$. Hence the net $\left\{\theta_{\pi}\right\}$ converges to $\delta_{e_{2}}$ in the weak*-topology of $X_{2}^{*}$. This implies that $\lambda\left(x_{\pi}\right) \rightarrow 1$ and the net $\delta_{\gamma\left(x_{n}\right)}$ converges to $\delta_{e_{2}}$ in the weak*-topology of $X_{2}^{*}$. Since $C_{0}\left(G_{2}\right) \subseteq X_{2}, \gamma\left(x_{\pi}\right)$ converges to $e_{2}$. Since $\left\{\gamma\left(x_{\pi}\right)\right\}$ is an arbitrary subnet of $\left\{\gamma\left(x_{2}\right)\right\}$, the net $\left\{\gamma\left(x_{\alpha}\right)\right\}$ must also converge to $e_{2}$. Therefore $\gamma$ is continuous. Similarly $\gamma^{-1}$ is also continuous. The theorem is established.

Remarks. 1. Theorem 1 yields Johnson's result [3, Corollary] when $X_{i}=C_{0}\left(G_{i}\right)$, $i=1,2$.

2. Theorem 1 is false without the hypothesis: $C_{0}\left(G_{1}\right) \subseteq X_{1}$, for example, if $G_{1}$ is any locally compact noncompact group, and $G_{2}$ is the set of multiplicative linear functionals on $\operatorname{AP}\left(G_{1}\right)$ with the Arens multiplication. Then $G_{2}$ is a compact group. Let $X_{1}=\operatorname{AP}\left(G_{1}\right)$ and $X_{2}=C\left(G_{2}\right)$. Then $X_{1} \cap C_{0}\left(G_{1}\right)=\{0\}$. Define $J: X_{1} \rightarrow X_{2}$ by $(J f)(\phi)=\phi(f)$ for each $f \in X_{1}, \phi \in G_{2}$. Then $J^{*}$ is an isometry and an algebra isomorphism from $X_{2}^{*}$ onto $X_{1}^{*}$.

\section{REFERENCES}

1. M. M. Day, Amenable semigroups, Illinois J. Math. 1 (1957), 509-544.

2. N. Dunford and J. T. Schwartz, Linear operators. I, Interscience, New York, 1958.

3. B. E. Johnson, Isometric isomorphisms of measure algebras, Proc. Amer. Math. Soc. 15 (1964), 186-188.

4. E. Hewitt and K. Ross, Abstract harmonic analysis, vol. 1, Springer-Verlag, Berlin, 1964.

5. Abstract harmonic analysis, vol. 2, Springer-Verlag, Berlin, 1970.

6. K. McKennon, Multipliers, positive functionals, positive-definite functions, and Fourier-Stieltjes transforms, Mem. Amer. Math. Soc., no. 111, 1971.

7. J. G. Wendel, Left centralizers and isomorphisms of group algebras, Pacific J. Math. 2 (1952), 251-261.

Department of Mathematics, University of Alberta, Edmonton, Alberta, Canada

Department of Mathematics, Washington State University, Pullman, Washington 99163 